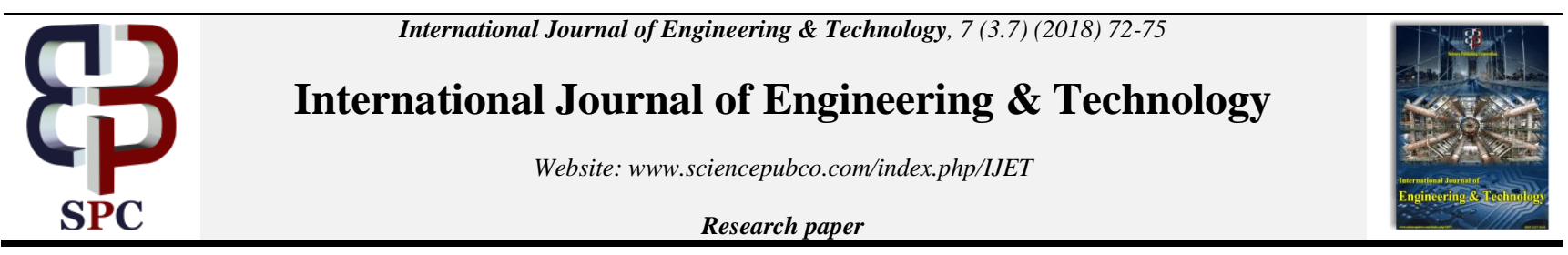

\title{
Clutter Distribution Analysis of A Tropical Foliage Clutter for Different Profiles Based on FSR Micro-Sensor Network
}

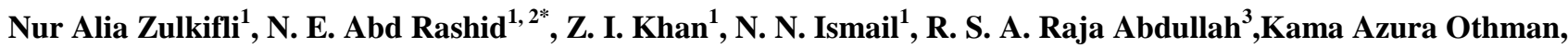 \\ Mahanijah Md Kamal \\ ${ }^{I}$ Applied Electromagnetic Research Group (AERG), ${ }^{2}$ Microwave Research Institute, \\ Universiti Teknologi Mara, 40450 Shah Alam, Selangor, Malaysia \\ ${ }^{2}$ Wireless and Photonic Networks Research Centre, Faculty of Engineering, Universiti Putra Malaysia (UPM), Serdang 43400, Malaysia \\ *Corresponding author E-mail: emileen98@salam.uitm.edu.my
}

\begin{abstract}
Comparison of four different clutter profiles (border, seaside, free space and forest) using Forward Scatter Radar (FSR), which operates in Ultra-High and Very High Frequency (UHF and VHF) bands, is analyzed in this paper. Clutter levels ranging from low, medium, strong and very strong on each profile were studied. Based on the standard deviation of each clutter profile, border suits the best profile as the strongest clutter profile amidst seaside and free space, while the forest is determined as the lowest clutter profile. Subsequently, the characteristics of the clutter are investigated and compared based on the five distribution models (Log-Normal, Log-Logistic, Gamma, Weibull and Nakagami). The parameters of the five distributions are evaluated using Root Mean Square Error (RMSE) in order to prove that the distribution model fits best to the clutter data. It can be concluded that Gamma distribution is the best distribution model for all cases of frequency bands and profiles.
\end{abstract}

Keywords: FSR; Wind Clutter; UHF/VHF; MLE; GOF; RMSE

\section{Introduction}

The idea of a wireless network for situational awareness based on FS sensors has been introduced in [1]. The system could detect and classifies moving target within the coverage area. In order to establish networks between sensors, the system utilizes omnidirectional antennas, hence it operates in a strong multipath environment and the absence of range resolution causes the clutter to collect from a larger volume around the FSR position.

Clutter is an unwanted echo from surrounding. It is usually masking target signal thus complicates the target detection and degrades the radar performance, which could give false alarm [2] Numerous researches have been conducted to understand and characterize clutter behavior, its distribution and model it Different factors such as wind, location, radar topology and frequency used which affect the clutter are studied in [3].

The measured clutter signal can be described probabilistically due to the fact that it is based on an uncertainty of the environment. There are numbers of distribution models that have been used to model the clutter signal in the literature especially for FSR. As reported in [4], Weibull distribution was used to model the clutter signal measured using FSR in dense woodland with VHF/UHF carrier frequency. In [5], ground clutter signal was measured in a dense forest and it was found that Log-Logistic model is the bestfitted model. Other areas such as open air, seaside and border are also investigated. However, to the best of the authors' knowledge, none of the works are focused on comparison of clutters from different places based on FSR. Therefore, in this paper, foliage clutter varies from open space to the dense environment, which involved forest, seaside, border and free space, which may affect the FSR performance is compared and investigated. Clutters were collected from different locations/profiles in order to observe its pattern and characteristics.

\section{Clutter Data Collection}

Considering the previous study [3], experiments have been conducted in four different locations with sensors baseline of $50 \mathrm{~m}$; measured up to 40 samples data in the form of RSSI signals. The experiments have taken place in:
i. Forest - a dense canopy planted forest,
ii. Seaside - beach area with less existence of variable height of trees,
iii. Border - area between the seaside and forest with less dense of woods,
iv. Free space area - empty parking space.

Clutter signals are taken at three different frequency bands, which are $64 \mathrm{MHz}, 151 \mathrm{MHz}$ and $434 \mathrm{MHz}$ simultaneously.

\section{Clutter Spectral Analysis}

Clutters are categorized based on its strength: low, medium, strong and very strong. In the previous research [3], wind speed has been used as the method to categorize the clutter; however this method is inaccurate due to the fact that the measurement of the wind speed was taken once before each data is collected. Furthermore, throughout 20 minutes of measurement, the wind speed can be 
fluctuated/varied. Therefore, in order to organize and categorize different strength of clutter, standard deviation (STD) of each clutter data is calculated based on the equation below:

$$
\sigma=\sqrt{\frac{1}{N} \sum_{I=1}^{N}\left(x_{i}-\bar{\mu}\right)^{2}}
$$

Subsequently, the clutter strength is divided into four different clutter strengths based on the equation in [3]:

$$
\text { STD }=\left(\frac{\text { hïghsTD walue-lowSTD walue }}{4}\right)+\text { Low STD value }
$$

Figure 1 displays the RSSI signals for the UHF and VHF frequencies measured at different four test sites for low clutter strength. As we can observe, the clutter increases in amplitude as the frequency escalates from 64 to $434 \mathrm{MHz}$. At $64 \mathrm{MHz}$ we can see that the lowest amplitude is $1.32 \mathrm{~V}$ (in the forest) and the highest amplitude is $1.82 \mathrm{~V}$ (at the border), while for frequency $151 \mathrm{MHz}$ verified the lowest and highest amplitude are $1.48 \mathrm{~V}$ and $1.72 \mathrm{~V}$ at Forest and Border, respectively. Contrarily, the highest amplitude is $1.79 \mathrm{~V}$ measured at a Border site for $434 \mathrm{MHz}$ while the lowest is $1.523 \mathrm{~V}$ at Forest. It can be seen is an increment in the amplitude as the frequency gets higher.

As tabulated in Table 1, RSSI level of the three frequency channels at forest recorded the lowest clutter compare to the other three locations and the strongest clutter was observed for border at higher frequencies; 151 and $434 \mathrm{MHz}$. The low clutter in the forest is due to the fact that the nature of the tropical forests itself, which has different heights of trees and a canopy layer (broadleaved evergreen trees). This may cause low penetration of wind from reaching the forest floor where the sensors were placed and minimized the swaying of the trees. In distinction, border is an area in between seaside and forest, which allows the swaying of wind more sturdy. Hence, strong magnitude of clutter signal could easily detect by the sensors.

Figure 2 demonstrates the example of Doppler signal for 64, 151 and $434 \mathrm{MHZ}$ respectively at border test site.
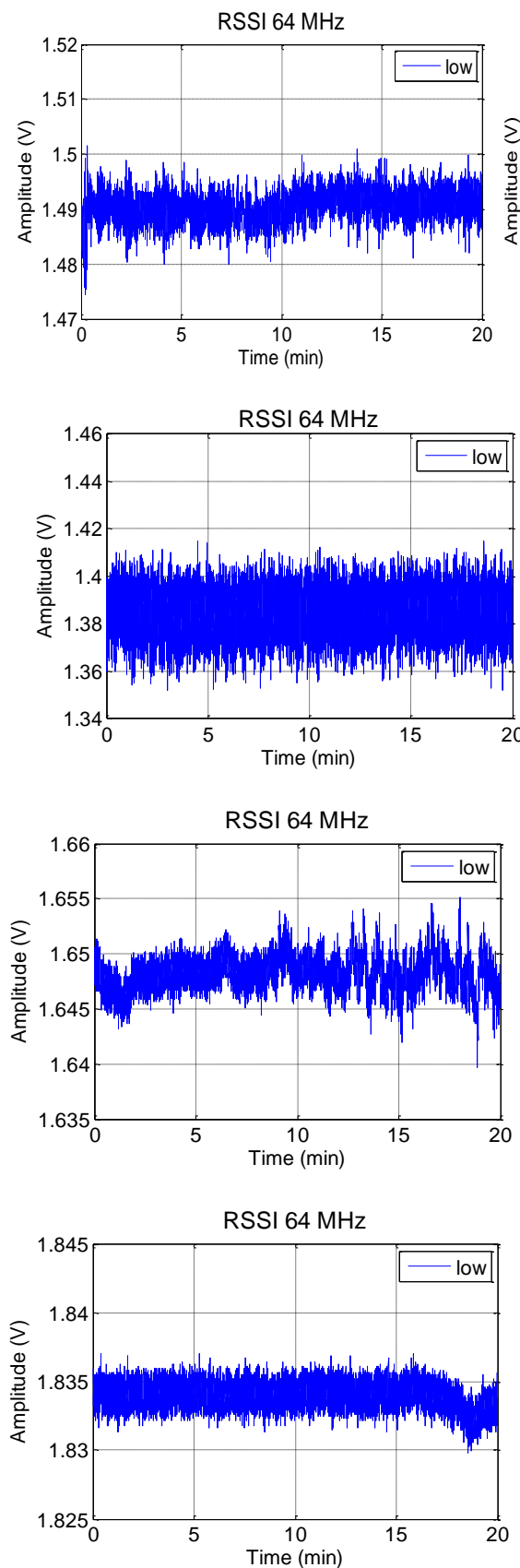

RSSI $151 \mathrm{MHz}$

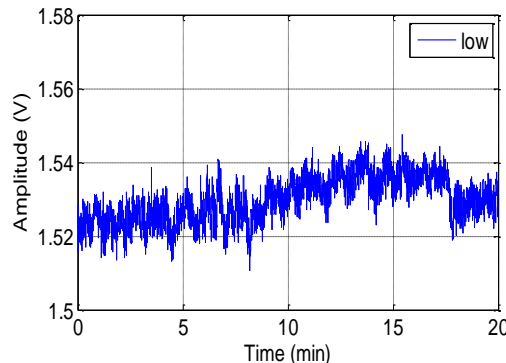

(a)

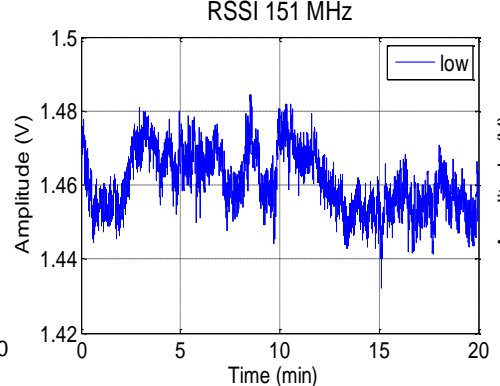

(b)

RSSI $151 \mathrm{MHz}$

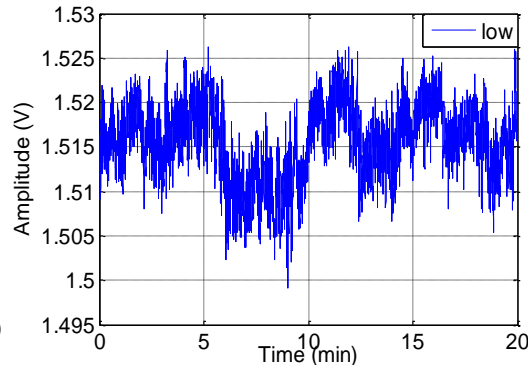

(c)

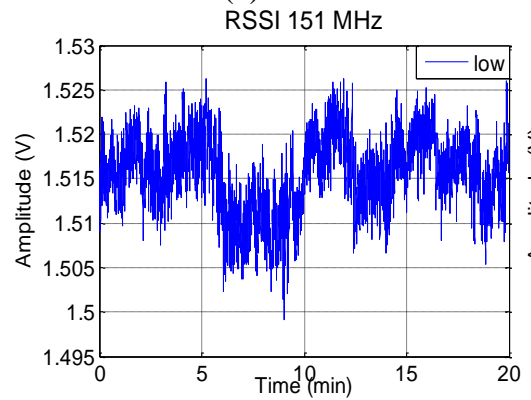

(d)

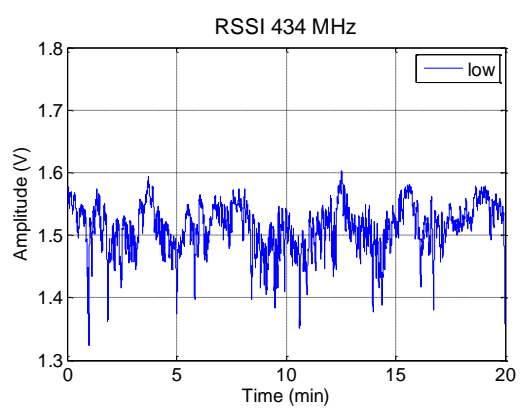

RSSI $434 \mathrm{MHz}$

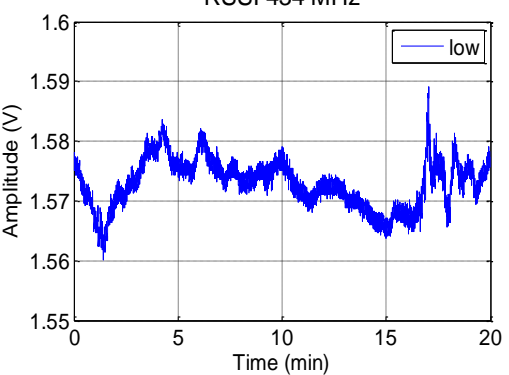

RSSI $434 \mathrm{MHz}$

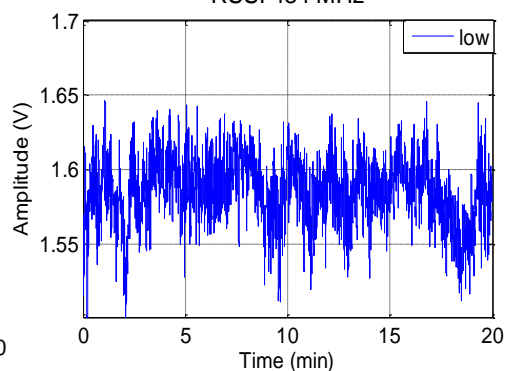

RSSI $434 \mathrm{MHz}$

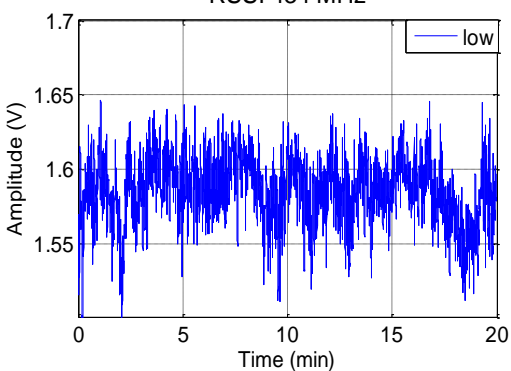

Figure 1: RSSI signals for (a) 64, (b) 151 and (c) $434 \mathrm{MHz}$ channel frequencies at low clutter for (a) seaside, (b) forest, (c) free space and (d) border. 

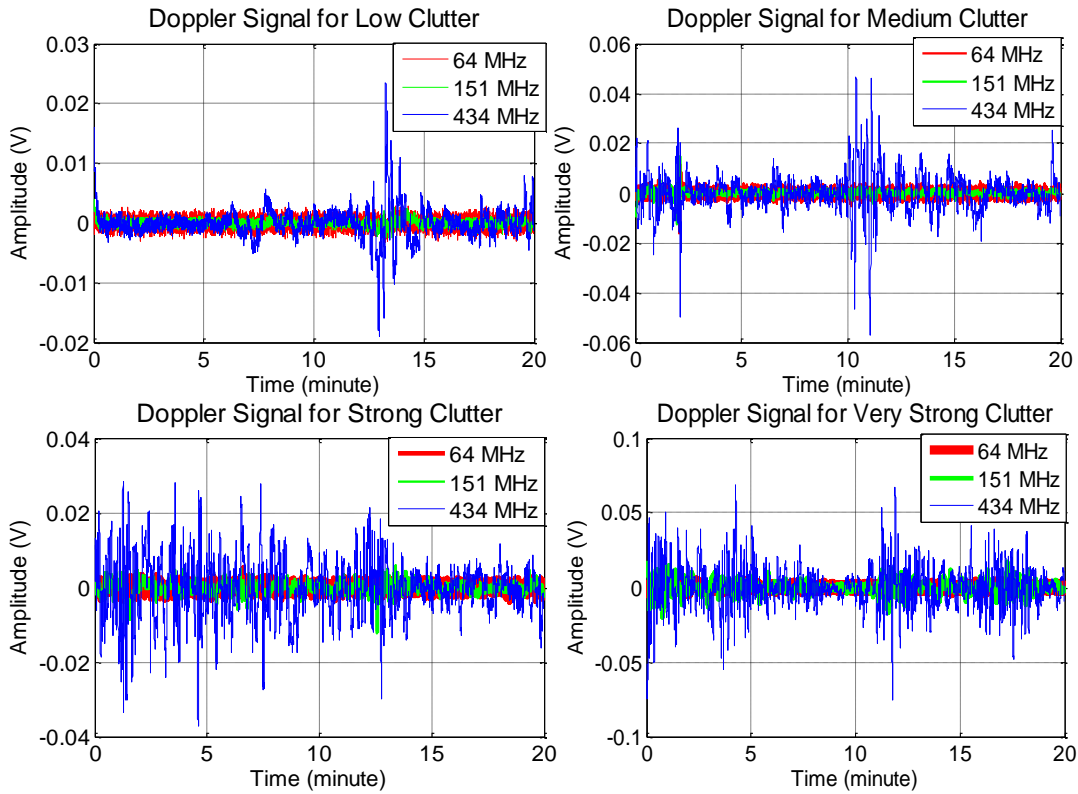

Figure 2: Doppler signals for 64, 151 and $434 \mathrm{MHz}$ channel frequencies at low, medium, strong and very strong clutter.

Table 1: Tabulated data for standard deviation value at seaside, forest, free space and border.

\begin{tabular}{|c|c|c|c|c|c|c|c|c|c|c|c|c|}
\hline \multirow{3}{*}{$\begin{array}{l}\text { Location } \\
\text { Clutter strength }\end{array}$} & \multicolumn{3}{|l|}{ Seaside } & \multicolumn{3}{|l|}{ Forest } & \multicolumn{3}{|c|}{ Free space } & \multicolumn{3}{|l|}{ Border } \\
\hline & \multicolumn{12}{|c|}{ Frequency (MHz) } \\
\hline & 64 & 151 & 434 & 64 & 151 & 434 & 64 & 151 & 434 & 64 & 151 & 434 \\
\hline Low & 0.0124 & 0.0132 & 0.0278 & 0.0151 & 0.0018 & 0.0044 & 0.0030 & 0.0001 & 0.0116 & 0.0005 & 0.0013 & 0.0080 \\
\hline Strong & 0.0158 & 0.0178 & 0.0346 & 0.0168 & 0.0026 & 0.0068 & 0.0157 & 0.0051 & 0.0422 & 0.0020 & 0.0023 & 0.0096 \\
\hline Very Strong & 0.0180 & 0.0201 & 0.0380 & 0.0319 & 0.0030 & 0.0080 & 0.0220 & 0.0071 & 0.0576 & 0.0027 & 0.0027 & 0.0104 \\
\hline
\end{tabular}

\section{Probability Density Function Models}

This section shows the clutter data with different distribution models in terms of the probability distribution function (PDF). The measured clutter signal is represented in a histogram form to show the probability of the clutter amplitude before the amplitude of the histogram is tested against the distribution models. Five distribution models as listed in Table 2 are used in this analysis.

Figure 3 shows the PDF comparisons between the clutter data of $64 \mathrm{MHz}, 151 \mathrm{MHz}$ and $434 \mathrm{MHz}$ with the distribution models for low, medium, strong and very strong clutter, respectively. From the figures, it can be clearly seen that Gamma model fits best to the clutter data for all levels of clutter strength.

In order to prove that the distribution model fits best to the clutter data based on the plot observation, goodness-of-fit (GOF) test is used in this analysis, which is a root mean square error (RMSE). The test is vital in calculating the error between the amplitude of measured clutter data and the amplitude of statistical model. The equation for RMSE is stated as [6]:

RMSE $=\sqrt{\frac{1}{n} \sum_{i=1}^{n}\left(c_{i}-\widehat{c}_{i}\right)^{2}}$

where the clutter data number of sample represents by $i$, clutter data amplitude value denoted by $\square \square$; while $\square \square$ is the statistical model's amplitude value.

The calculated errors for clutter signal $64 \mathrm{MHz}, 151 \mathrm{MHz}$ and 434 $\mathrm{MHz}$ using RMSE are tabulated in Table 3. From the table, the RMSE for Gamma model record the smallest error for all frequencies as compared to the other four models, indicating that it is the best model that fits the clutter signal.

Table 2: Distribution model parameters

\begin{tabular}{|c|c|c|}
\hline Distribution & Equation & Parameters \\
\hline Log-Normal & $f(x \| \mu, \sigma)=\frac{1}{x \sigma \sqrt{2 \pi}} \exp \left[\frac{-(\ln x-\mu)^{2}}{2 \sigma^{2}}\right.$ & $\begin{array}{l}\mu=\text { location } \\
\sigma=\text { scale }\end{array}$ \\
\hline Log logistic & $f(x \| \mu, \sigma)=\frac{1}{\sigma} \frac{1}{x} \frac{\theta^{2}}{\left(1+e^{2}\right)^{2}}$ & $\begin{array}{l}\mu=\text { location } \\
\sigma=\text { scale }\end{array}$ \\
\hline Gamma & $f(x \mid a, b)=\frac{1}{b^{a} \Gamma(a)} x^{a-1} \in \frac{a x}{b}$ & $\begin{array}{l}a=\text { shape } \\
b=\text { scale }\end{array}$ \\
\hline Weibull & 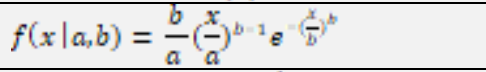 & $\begin{array}{l}a=\text { scale } \\
b=\text { shape }\end{array}$ \\
\hline Nakagami & $f(x \| \mu, \omega)=2\left(\frac{\mu}{\omega}\right)^{\mu} \frac{1}{(\mu)} x^{3 \mu-1} e^{\frac{\omega \mu}{\omega} x^{2}}$ & $\begin{array}{l}\mu=\text { shape } \\
\sigma=\text { scale }\end{array}$ \\
\hline
\end{tabular}




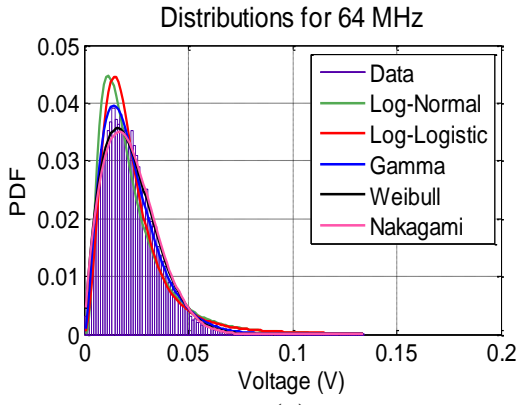

(a)

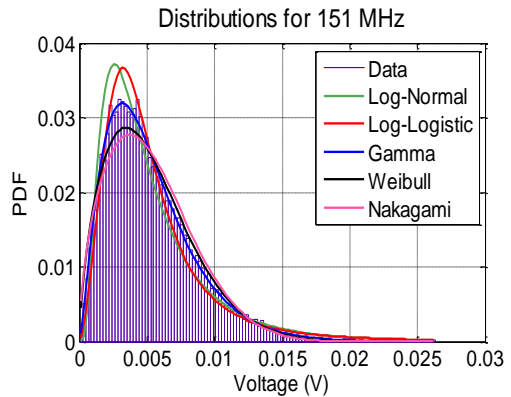

(b)

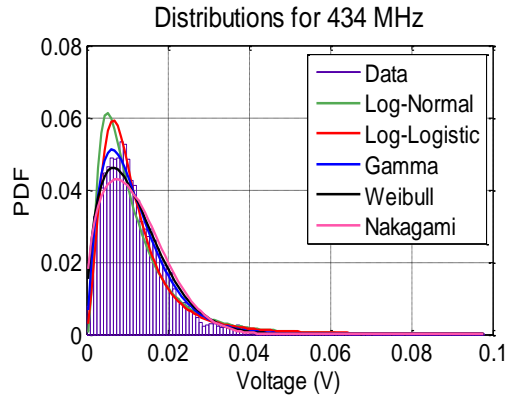

(c)

Figure 3: Comparison of distribution models for (a) $64 \mathrm{MHz}$, (b) $151 \mathrm{MHz}$ and (c) $434 \mathrm{MHz}$

Table 3: Average estimated RMSE.

\begin{tabular}{|l|l|l|l|l|l|}
\hline \multirow{2}{*}{ Frequency } & Distribution Models & Gog-Logistic & Gamma & Weibull \\
\cline { 2 - 6 } & Log-Normal & Nakagami & 0.0024 \\
\hline $64 \mathrm{MHz}$ & 0.0017 & 0.0019 & 0.0016 & 0.0038 \\
\hline $151 \mathrm{MHz}$ & 0.0034 & 0.0022 & 0.0016 & 0.027 & 0.0031 \\
\hline $434 \mathrm{MHz}$ & 0.0032 & 0.0020 & 0.0015 & 0.030 & \\
\hline
\end{tabular}

\section{Conclusion}

This paper shows the comparison of four different ground clutter profiles based on FSR Micro-Sensor Network at $64 \mathrm{MHz}, 151$ $\mathrm{MHz}$ (VHF) and $434 \mathrm{MHz}$ (UHF). The clutter data were collected at the border of a dense forest, seaside, forest and a free space area. The analysis was done by calculating the standard deviation of the clutter and categorizes the clutter signal to four levels clutter strength. From the analysis, border is chosen as the strongest area of clutter while forest as the lowest clutter strength area.

Histogram of different five types of distribution which are LogLogistic, Log-Normal, Gamma, Weibull and Nakagami demonstrated the modelling of the clutter signal tested in this experiment. Comparing the five distributions model, Gamma is chosen as the accurate distribution model. GOF test (by calculating RMSE) proved the Gamma distribution model as the most accurate for 64 , 151 and $434 \mathrm{MHz}$ frequency band, as the lowest error recorded.

\section{Acknowledgement}

This work was supported by the Institute of Research Management and Innovation (IRMI), Universiti Teknologi MARA (Grant no: 600-RMI/DANA 5/3/LESTARI (78/2015)) of Universiti Teknologi MARA.

\section{References}

[1] M. Antoniou, V. Sizov, C. Hu, P. Jancovic, R. Abdullah, N. E. A Rashid, and M. Cherniakov, The Concept of A Forward Scattering Micro-Sensors Radar Network for Situational Awareness, in Proceedings of The 2008 International Conference on Radar, 2008.

[2] N. E. A. Rashid, P. Jancovic, M. Gashinova, M. Cherniakov, and V. Sizov, The Effect of Clutter on The Automatic Target Classification Accuracy in FSR, in IEEE National Radar Conference, 2010.

[3] N. A. Zakaria, M. Cherniakov, M. Gashinova, and V. Sizov, Empirical Clutter Analysis for Forward Scatter Micro-Sensors, in 15th International Radar Symposium, 2014.

[4] N. N. Ismail, N. E. A. Rashid, Z. I. Khan, and M. F. A. Rashid, Measurement, Processing and Modeling of A Tropical Foliage Clutter Using Forward Scatter Radar Micro-Sensor Network with VHF and UHF Bands, in 2015 International Conference on Radar, Antenna, Microwave, Electronics and Telecommunications, 2015.

[5] V. Sizov, M. Gashinova, N. E. A. Rashid, J. Chen, and M. Cherniakov, Forward Scattering Micro Radar Efficiency Analysis for Different Landscapes, in 6th EMRS DTC Technical Conference, 2009.

[6] N. N. Ismail, N. E. A. Rashid, N. A. Zakaria, Z. I. Khan, and V. Sizov, Clutter Modeling for Forward Scatter Radar (FSR) Micro-
Sensor Network with Ultra High Frequency (UHF) Band, in IEEE 11th International Colloquium on Signal Processing \& its Applications, 2015. 